\title{
On the fluid-fluid phase separation in charged-stabilized colloidal suspensions
}

\author{
Yan Levin $^{1,2}$, Emmanuel Trizac ${ }^{2}$ and Lydéric Bocquet ${ }^{3}$ \\ ${ }^{1}$ Instituto de Física, Universidade Federal do Rio Grande do Sul, Caixa Postal 15051, \\ CEP 91501-970, Porto Alegre, RS, Brazil \\ ${ }^{2}$ Laboratoire de Physique Théorique, UMR CNRS 8627, Université de Paris XI, Bâtiment 210, \\ F-91405, Orsay Cedex, France \\ 3 LPMCN, UMR CNRS 5586, Université Claude Bernard Lyon 1, 43 boulevard du 11 novembre \\ 1918, 69622 Villeurbanne Cedex, France
}

E-mail: levin@if.ufrgs.br, Emmanuel.Trizac@th.u-psud.fr and

Lyderic.Bocquet@1pmcn.univ-lyon1.fr

Received 18 July 2003

Published 20 November 2003

Online at stacks.iop.org/JPhysCM/15/S3523

\begin{abstract}
We develop a thermodynamic description of particles held at a fixed surface potential. This system is of particular interest in view of the continuing controversy over the possibility of a fluid-fluid phase separation in aqueous colloidal suspensions with monovalent counterions. The condition of fixed surface potential allows one in a natural way to account for the colloidal charge renormalization. In a first approach, we assess the importance of the so called 'volume terms' and find that in the absence of salt, charge renormalization is sufficient to stabilize the suspension against a fluid-fluid phase separation. The presence of salt, on the other hand, is found to lead to an instability. A very strong dependence on the approximations used, however, puts the reality of this phase transition in serious doubt. To further understand the nature of the instability we next study a jellium-like approximation, which does not lead to a phase separation and produces a relatively accurate analytical equation of state for deionized suspensions of highly charged colloidal spheres. A critical analysis of various theories of strongly asymmetric electrolytes is presented, to assess their reliability as compared to the Monte Carlo simulations.
\end{abstract}

\section{Introduction}

There is a long standing debate in the field of colloidal science concerning the possibility of phase separation in aqueous colloidal suspensions containing monovalent counterions [1-14]. Charged colloids provide a particular challenge to the theorists. The long ranged Coulomb interaction and the extremely large asymmetry between the polyions and the counterions makes it very difficult to apply to this system the traditional methods of the liquid state theory. In particular, such a well established tool of condensed matter theorists as integral equations is 
found to be useless when applied to strongly asymmetric electrolytes at large couplings (low temperatures). For a wide range of parameters of physical interest the integral equations fail to even converge. The original hope of associating the lack of convergence with an underlying physical instability has proven to be unfounded $[15,16]$.

Recently, a linearized density functional theory has been used to study charged colloidal suspensions $[1,4]$. The theory predicted a thermodynamic instability which manifested itself as a fluid-fluid phase separation. However, the underlying approximation of the theory put its conclusions in doubt [7-9]. Specifically the linearization of the density functional lowers the statistical weight of the configurations in which the counterions are in the close vicinity of the polyions. This effect can be partially accounted for through the renormalization of the bare colloidal charge $[17,18]$. Unlike the bare charge, which can be very large, the effective (renormalized) charge is found to be bounded by the saturation value controlled-in a given solvent-by the colloidal size, temperature and salt concentration. The renormalization of colloidal charge was argued to wash out the phase transition predicted by the linear theories [7]. Furthermore, numerical solutions of the full non-linear Poisson-Boltzmann (PB) equation inside a Wigner-Seitz (WS) cell show the absence of any instability $[8,9,12]$. On the other hand, linearization of the PB equation leads to a non-convex pressure, as a function of colloidal concentration, similar to the one observed in other linear theories. All these suggest that the phase instability predicted by the linear theories might be an artifact of the underlying approximations.

To further explore these interesting points, we have investigated the thermodynamics of particles fixed at constant surface potential $[19,20,22]$. The relevance of the constant potential ensemble follows from the observation that unlike the bare charge, the effective charge of colloidal particles does not grow indefinitely but instead saturates. The saturation value is such that the effective electrostatic potential $\phi_{\mathrm{s}}$ in the vicinity of colloidal surface is $\beta q \phi_{\mathrm{s}} \approx 4$, where $\beta=1 / k_{\mathrm{B}} T$ and $q$ is the elementary charge. Thus, although the colloidal charge can be very large, the potential near the colloidal surface, i.e. within the Debye length, does not increase beyond $\beta q \phi_{\mathrm{s}} \approx 4$. This simple observation is sufficient for constructing a consistent thermodynamic description of colloids with a state dependent effective charge. Since the WS cell description of a colloidal suspension [21] does not lead to a fluid-fluid phase separation (see [12] for a general argument), to further understand the mechanism of the instability observed within the linearized theories, we focus on this alternative treatment.

We shall first (section 2) study the effect of charge renormalization on the polyionmicroion interaction free energy, i.e. the volume term that appears when the original mixture of colloids, coions and counterions is mapped onto an effective one component system of dressed colloids [1, 2, 4]. In section 3 an alternative derivation of the thermodynamic equation of state based only on the far field considerations is presented. The corresponding pressure-density isotherms, in this case, do not exhibit criticality at any salt concentration. In order to assess the reliability of various approaches, in section 4 we compare the corresponding pressures to the results of the Monte Carlo (MC) simulations of Linse [6]. We also consider the, recently proposed, symmetric PB [10] and the 'bootstrap' PB [11] theories. Conclusions are drawn in section 5.

\section{Role of volume terms}

\subsection{State dependent effective charges}

Consider a colloidal suspension at concentration $\rho_{\mathrm{p}}$, containing spherical polyions of charge $-Z q$ and radius $a$ in contact with a monovalent salt reservoir at concentration $c_{\mathrm{s}}$. Now suppose 
one colloid is fixed at $r=0$. In a continuum approximation consisting of smearing out the charge of other colloids and linearizing the PB equation [22], the electrostatic potential at distance $r$ from the centre of the colloid is

$$
\phi(r)=-\frac{Z q \theta(\kappa a) \mathrm{e}^{-\kappa r}}{\epsilon r}, \quad \theta(x)=\frac{\mathrm{e}^{x}}{(1+x)},
$$

where the inverse Debye length is

$$
\kappa=\sqrt{4 \pi \lambda_{\mathrm{B}}\left(\rho_{+}+\rho_{-}\right)}
$$

and the Bjerrum length is

$$
\lambda_{\mathrm{B}}=\frac{q^{2}}{\epsilon k_{\mathrm{B}} T} \text {. }
$$

The mean densities of coions and counterions inside the suspension are respectively $\rho_{-}$and $\rho_{+}$. When $\rho_{\mathrm{p}} \rightarrow 0, \kappa^{2} \rightarrow \kappa_{\mathrm{s}}^{2}=8 \pi \lambda_{\mathrm{B}} c_{\mathrm{s}}$.

For highly charged polyions, equation (1) strongly overestimates the real electrostatic potential. However, it can be made consistent with the full non-linear PB theory, if instead of the bare charge $Z$ an effective, renormalized, charge $Z_{\text {eff }}$ is used. The observation that for large surface potentials the electrostatics away from the colloidal surface is completely insensitive to the surface charge density allows for the 'far field' definition of the effective charge. Specifically, viewed from a distance larger than the Debye length and provided that $\kappa a>1$, the surface potential of a strongly charged colloidal particle appears to be $\beta q \phi(a) \approx-4[19,20,22]$. Combining this with equation (1) leads directly to [19, 22]

$$
Z_{\mathrm{eff}}=\frac{4 a}{\lambda_{\mathrm{B}}}(1+\kappa a)
$$

We should note that this is the saturated value of the effective charge relevant for the highly charged colloidal particles. For weakly charged particles there is little or no charge renormalization. In the infinite dilution limit [23] of one colloid immersed in a 1:1 electrolyte of concentration $c_{\mathrm{s}}$, the exact result for the saturation limit of $Z_{\mathrm{eff}}$ is

$$
Z_{\mathrm{eff}}=\frac{4 a}{\lambda_{\mathrm{B}}}\left(\frac{3}{2}+\kappa_{\mathrm{s}} a\right)
$$

This expression — valid up to corrections of order $(\kappa a)^{-1}$, which turn out to be quite small as soon as $\kappa a>1$-is very close to the approximation (4), derived from the matching procedure detailed in [20, 22].

The effective charge depends strongly on the electrolyte concentration inside the suspension. Salt screens the electrostatic interactions between the counterions and the polyions and leads to an increase in the colloidal effective charge. While the effective charge of colloidal particles is a strongly state dependent function, the effective surface potential is not. Therefore, inside a suspension, colloids behave as if their surface potential was effectively fixed. It is interesting, therefore, to study the thermodynamics of colloidal particles at fixed surface potential.

\subsection{Thermodynamics of particles at fixed surface potential}

The change of the thermodynamic ensemble from the constant charge to the constant surface potential allows us in a natural way to explore the role of charge renormalization in the framework of a linear theory. The surface potential is related to the effective colloidal charge through the equation (1)

$$
\varphi=-\beta q \phi(a)=\frac{Z \lambda_{\mathrm{B}}}{a(1+\kappa a)} .
$$


As mentioned above, the reduced surface potential within the non-linear PB theory is found to saturate at $\varphi=4$. For the sake of generality we shall, however, keep its value arbitrary. In the subsequent analysis, $Z$ will refer to the saturation value of the colloidal effective charge. An implicit assumption is therefore that the bare charge substantially exceeds the effective one.

In the simplest approximation, the Helmholtz free energy of the suspension is a sum of entropic and electrostatic contributions:

$$
\beta F=N_{+}\left[\ln \rho_{+} \Lambda^{3}-1\right]+N_{-}\left[\ln \rho_{-} \Lambda^{3}-1\right]+\beta F^{\mathrm{el}},
$$

where $\Lambda$ is the de Broglie thermal wavelength and $N_{+}$and $N_{-}$refer to the numbers of counterions and coions inside the suspension.

The electrostatic free energy results from the polyion-microion, microion-microion and polyion-polyion interactions. For suspensions containing monovalent counterions the polyion-counterion interaction is the dominant contribution and will be the only one kept in the present exposition (we come back to this point in section 4). We find [22]

$$
\beta F^{\mathrm{el}}=\frac{Z^{2} \lambda_{\mathrm{B}} N_{\mathrm{p}}}{2 a(1+\kappa a)} .
$$

This expression can be obtained through the usual Debye charging process in which all the particles are simultaneously charged from 0 to the their final charge [22]. Alternatively a surface charging process, at constant Debye length, can be employed [24]. It is noteworthy that in the salt free case, we recover precisely the volume term obtained in $[1,4]$.

If the suspension is in contact with a salt reservoir of chemical potential $\mu_{\mathrm{s}}$, the effective charge of colloidal particles, as well as the number of counterions and coions, is determined by the minimum of the grand potential function

$$
\begin{aligned}
& \beta \Omega=\beta F-\beta \mu_{\mathrm{s}}\left(N_{+}+N_{-}\right)-(\varphi+\gamma) Z N_{\mathrm{p}} \\
& -\omega N_{\mathrm{p}}\left[\varphi(1+\kappa a)-\frac{Z \lambda_{\mathrm{B}}}{a}\right]-\gamma\left(N_{+}-N_{-}-Z N_{\mathrm{p}}\right) .
\end{aligned}
$$

In this equation, $\omega$ and $\gamma$ are the Lagrange multipliers: $\gamma$ ensures charge neutrality of the system, while $\omega$ enforces the relationship between the surface potential and the effective charge, equation (6). In the biophysics literature $\gamma$ is known as the Donnan potential. It results from the inability of macroions to diffuse through a semi-permeable membrane. This is precisely the situation that we have in mind; while the microions are assumed to be in free exchange with the reservoir, the polyions are confined to the interior of the suspension. This restriction on the polyion mobility results in a potential difference $\gamma$, between the bulk of the suspension and the reservoir. The colloids are then held at potential $\varphi+\gamma$ with respect to the reservoir, or equivalently at potential $\varphi$ with respect to the bulk of the suspension. Within the WS cell model, a similar prescription for constraining the potential difference between the colloidal surface and the outer (reservoir) boundary has been shown to yield a surprisingly good agreement with the full non-linear PB equation [20].

Minimizing the grand potential with respect to $N_{+}, N_{-}$and $Z$, we find

$$
\begin{aligned}
& \frac{\partial \beta \Omega}{\partial N_{ \pm}}=\frac{\partial \beta F}{\partial N_{ \pm}}-\mu_{\mathrm{s}}-\omega\left[\frac{2 \pi \varphi \lambda_{\mathrm{B}} a \rho_{\mathrm{p}}}{\kappa}\right] \mp \gamma=0, \\
& \frac{\partial \beta \Omega}{\partial Z}=\frac{\partial \beta F}{\partial Z}-\omega N_{\mathrm{p}}\left[\frac{\lambda_{\mathrm{B}}}{a}\right]-\varphi N_{\mathrm{p}}=0
\end{aligned}
$$

and the charge neutrality condition reads

$$
N_{+}-N_{-}=\frac{\varphi a(1+\kappa a)}{\lambda_{\mathrm{B}}} N_{\mathrm{p}} .
$$


Noting that

$$
\frac{\partial \beta F}{\partial Z}=\varphi N_{\mathrm{p}},
$$

equation (11) simplifies to

$$
\omega=0 .
$$

Eliminating the Lagrange multiplier between equations (10), we are left with two equations which govern the concentrations of counterions and coions inside the suspension,

$$
\rho_{+} \rho_{-}=c_{\mathrm{s}}^{2} \exp \left(\frac{2 \pi \varphi^{2} a^{2} \rho_{\mathrm{p}}}{\kappa}\right)
$$

and

$$
\rho_{+}-\rho_{-}=\frac{\varphi a(1+\kappa a)}{\lambda_{\mathrm{B}}} \rho_{\mathrm{p}}
$$

\subsection{The equation of state}

The osmotic pressure inside the suspension of colloids at fixed surface potential is

$$
P=-\left.\frac{\mathrm{d} \Omega}{\mathrm{d} V}\right|_{N_{\mathrm{p}}, \mu_{\mathrm{s}}, \omega, \gamma, \varphi} .
$$

It is important to keep in mind that as $\Omega$ changes with volume; the number of coions, that of counterions, as well as the charge of colloidal particles are all varying. This is the reason for writing the total derivative in the expression (17):

$$
\left.\frac{\mathrm{d} \Omega}{\mathrm{d} V}\right|_{N_{\mathrm{p}}, \mu_{\mathrm{s}}, \omega, \gamma, \varphi}=\frac{\partial \Omega}{\partial N_{+}} \frac{\mathrm{d} N_{+}}{\mathrm{d} V}+\frac{\partial \Omega}{\partial N_{-}} \frac{\mathrm{d} N_{-}}{\mathrm{d} V}+\frac{\partial \Omega}{\partial Z} \frac{\mathrm{d} Z}{\mathrm{~d} V}+\frac{\partial \Omega}{\partial V} .
$$

Recalling that at the thermodynamic equilibrium

$$
\frac{\partial \Omega}{\partial N_{+}}=\frac{\partial \Omega}{\partial N_{-}}=\frac{\partial \Omega}{\partial Z}=0
$$

the expression for pressure simplifies to

$$
P=-\left.\frac{\partial \Omega}{\partial V}\right|_{N_{\mathrm{p}}, N_{+}, N_{-}, Z, \mu_{\mathrm{s}}, \omega, \gamma, \varphi}=-\left.\frac{\partial F}{\partial V}\right|_{N_{\mathrm{p}}, N_{+}, N_{-}, Z} .
$$

Equation (20) beautifully illustrates the thermodynamic principle of ensemble equivalence. The functional form of the pressure is the same whether the calculation is done in the fixed potential ensemble using the grand potential function $\Omega$, or in the fixed colloidal charge ensemble using the Helmholtz free energy $F$. We stress that simply inserting $Z_{\text {eff }}(V)$ with its state dependence into $F$ and then differentiating it with respect to volume will lead to an incorrect result. If the Helmholtz free energy is used, the variation must be performed at fixed colloidal charge.

In general it can be very difficult to find a suitable thermodynamic potential for a constrained system. The calculation of pressure, on the other hand, can be done very straightforwardly using the constant $Z$ ensemble and enforcing the constraint a posteriori. Evaluating the partial derivative in equation (20), the osmotic pressure inside the suspension takes a particularly simple form:

$$
\beta P=\rho_{+}+\rho_{-}-\frac{1}{4} \frac{a}{\lambda_{\mathrm{B}}} \varphi^{2} \kappa a \rho_{\mathrm{p}}
$$


where the concentrations of coions and counterions are determined from equations (15) and (16).

In the special case of vanishing salt concentration, equations (15) and (16) simplify to

$$
\begin{aligned}
& \rho_{-}=0, \\
& \rho_{+}=\varphi \rho_{\mathrm{p}} \frac{a}{\lambda_{\mathrm{B}}}\left[1+\frac{3}{2} \eta \varphi+\frac{1}{2} \sqrt{3 \eta \varphi(4+3 \eta \varphi)}\right]
\end{aligned}
$$

and the ratio of colloidal size to Debye length is

$$
\kappa a=\frac{3 \eta \varphi}{2}+\frac{1}{2} \sqrt{3 \eta \varphi(4+3 \eta \varphi)},
$$

where $\eta=4 \pi \rho_{\mathrm{p}} a^{3} / 3$ is the macroion volume fraction. For salt free suspensions the pressure becomes

$$
4 \pi \lambda_{\mathrm{B}} a^{2} \beta P=3 \eta \varphi\left(1+\kappa a-\frac{1}{4} \varphi \kappa a\right) .
$$

If $\varphi<4$ the osmotic pressure is a convex up function of colloidal density. For real colloids with $\varphi=4$, the pressure is a linear function of colloidal density, $\beta P=4 \rho_{\mathrm{p}} a / \lambda_{\mathrm{B}}$. For surface potentials strictly above 4 , a thermodynamic instability appears. It is very curious to note that the instability sets in precisely at $\varphi=4$, which is the saturation value for the surface potential obtained within the non-linear PB theory. Renormalization of the electrostatic free energy is, therefore, sufficient to stabilize a salt free real colloidal suspension $(\varphi=4)$ against a fluidfluid phase separation [7]. However, the fact that the critical surface potential is precisely equal to the saturation value of the non-linear theory suggests that the approach is most probably very sensitive to the approximations made. Furthermore, a relatively small amount of salt destabilizes the suspension even when $\varphi=4$; see figure 1 . The critical salt concentration for highly charged colloids of radius $a=1000 \AA$ is $c_{\mathrm{s}}^{*} \approx 10^{-4} \mathrm{M}$, which corresponds to $\kappa_{\mathrm{s}}^{*} a \simeq 3.3$. Since there is no explicit polyion-polyion or microion-microion interaction, the instability is completely driven by the polyion-counterion correlations. In figure 2 we show the effective charge $Z$ resulting from our approach, as a function of the volume fraction. A good agreement with the PB cell model is found.

It is important to stress that even a minor modification of the approximations employed may have a dramatic effect on the predicted phase instability. One may wish for instance to use equation (5) for the effective charge instead of equation (4). It is important to note, however, that such a modification means that colloid is no longer held at fixed potential. Therefore, the grand potential $\Omega$, as written in (9), can no longer be used. However, we can compute the functional dependence of $P$ on $Z, \rho_{\mathrm{p}}$ and $c_{\mathrm{s}}$, by differentiating the Helmholtz free energy $F$ with respect to volume at constant $Z$ and enforcing the constraint $Z=a(4 \kappa a+6) / \lambda_{\mathrm{B}}$ a posteriori. Following this route, we recover the same equation of state as before (i.e. equation (25) in the salt free case but with now a different salt dependence of $Z$ ) and the critical salt concentration above which the instability sets in (see figure 3) decreases by a factor of four to $\kappa_{\mathrm{s}}^{*} a \simeq 1.77$.

\section{An alternative approach: a jellium approximation}

In the previous section, we have found a fairly accurate expression for the effective colloidal charge at saturation and used it to renormalize the electrostatic free energy. It is important to remember, however, that the effective charge is by definition related to the 'far field' asymptotic properties of the electrostatic potential. Its use for the renormalization of electrostatic free energy is, therefore, questionable, since not only the far field but also the near field properties of the electrostatic potential may be relevant. We now turn our attention to a simple approach which relies only on the far field features of the electrostatic potential for obtaining the equation 


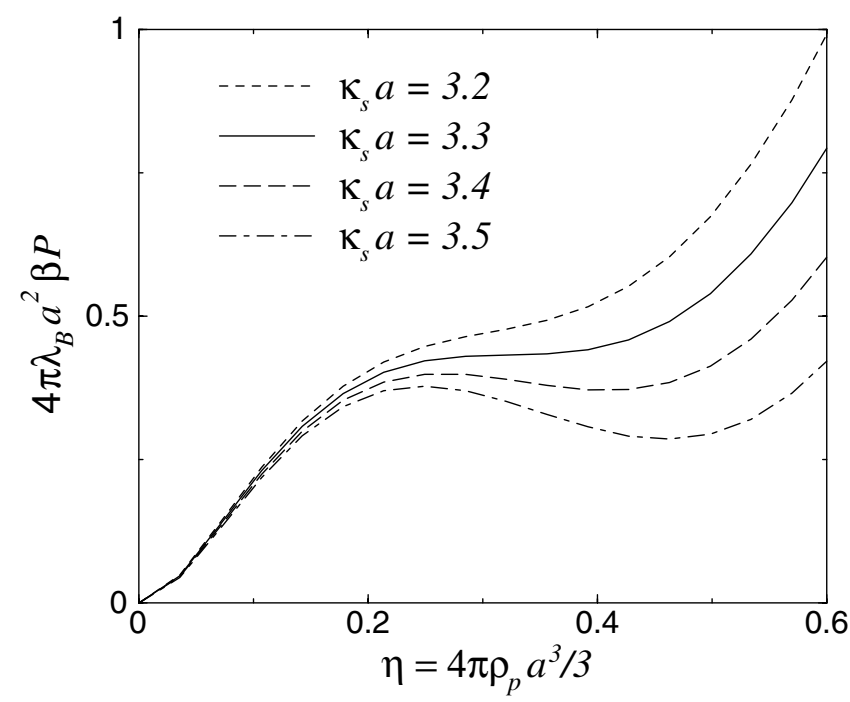

Figure 1. Pressure isotherms for different reservoir salt concentrations $\left(\kappa_{\mathrm{s}}^{2}=8 \pi \lambda_{\mathrm{B}} c_{\mathrm{s}}, \varphi=4\right)$.

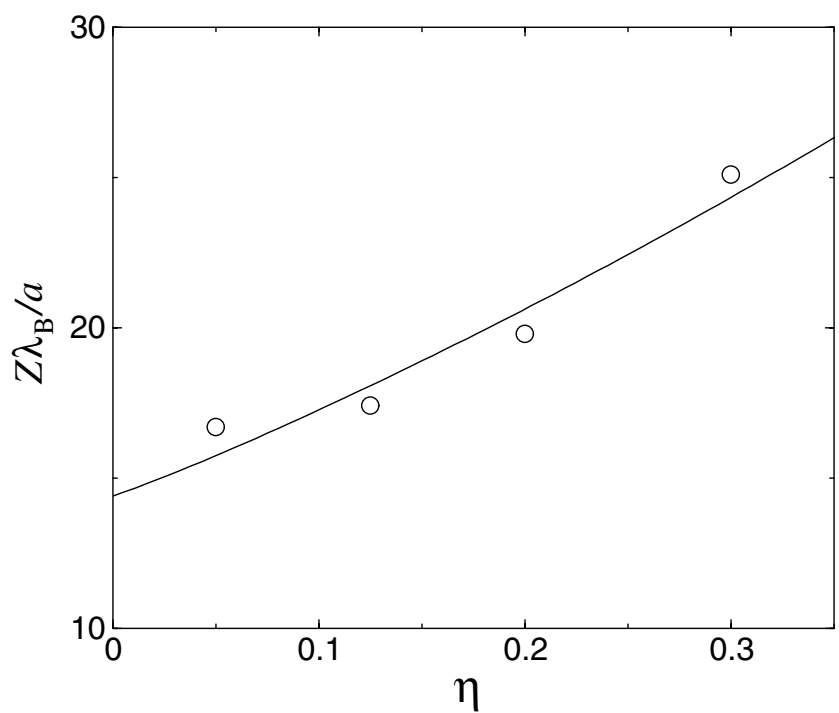

Figure 2. Comparison between the effective charge (solid curve) found using our variational approach and that obtained within the PB cell theory (circles), following the prescription proposed by Alexander et al [18, 34].

of state. As within the WS cell picture, use will be made of the contact theorem which relates the osmotic pressure to the concentration of counterions in the region where the electric field is zero [21].

We now reconsider the approach put forward at the beginning of section 2. Consider one colloidal particle fixed at the origin of the coordinate system. As before the charge of other microions is uniformly smeared throughout the solution. On the other hand, positions of counterions are strongly correlated with those of colloids. The system then forms a jellium, 


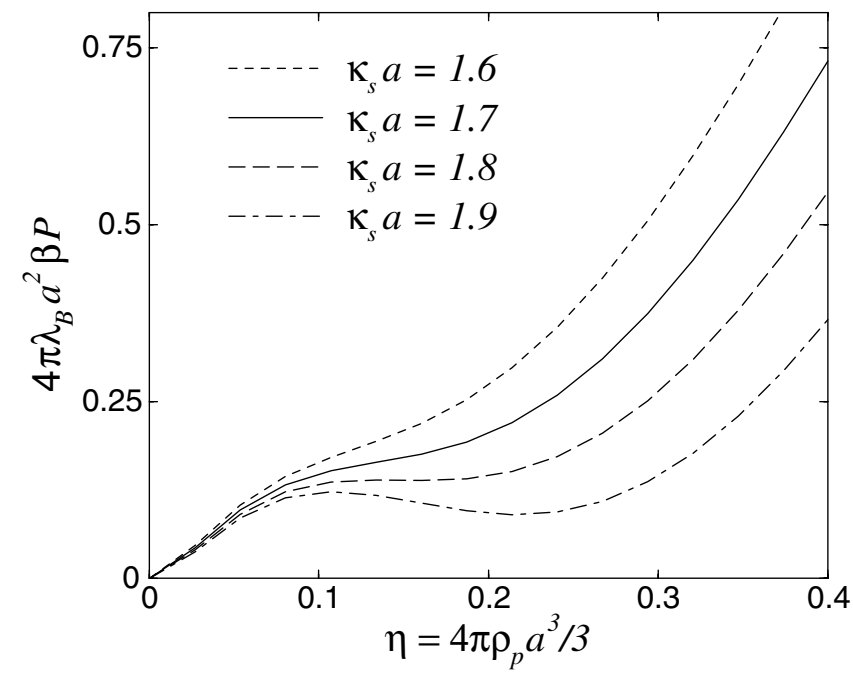

Figure 3. Pressure isotherms for different reservoir salt concentrations, making use of equation (5) instead of equation (4). The situation is now different from that of the previous constant $\varphi$ ensemble. Working is the constant $Z$ ensemble with the Helmholtz free energy (7) nevertheless allows one to compute the pressure.

where the electrostatic potential far from the colloid $\phi_{\infty}$ (for the bulk) differs from that in the reservoir (chosen to vanish). The solution of the linearized PB equation for $\delta \phi=\phi-\phi_{\infty}$ is again given by equation (1), where the screening length is related to the bulk salt concentration, $\kappa^{2}=4 \pi \lambda_{\mathrm{B}}\left(\rho_{+}(\infty)+\rho_{-}(\infty)\right)$. In the spirit of the previous discussion, the colloidal particles are held at constant surface potential $\varphi=4$ with respect to the bulk $\phi_{\infty}$, which again imposes $Z=4 a(1+\kappa a) / \lambda_{\mathrm{B}}$. The counterions and coions are distributed inside the jellium in accordance with the Boltzmann distribution

$$
\begin{aligned}
& \rho_{+}(r)=c_{\mathrm{s}} \mathrm{e}^{-\beta q \phi(r)}, \\
& \rho_{-}(r)=c_{\mathrm{s}} \mathrm{e}^{+\beta q \phi(r)},
\end{aligned}
$$

where $\phi(r)$ is the local electrostatic potential with respect to the reservoir. Taking the product of equations (26) and (27) we find the familiar condition for Donnan equilibrium $\rho_{+}(r) \rho_{-}(r)=c_{\mathrm{s}}^{2}$. The electroneutrality constraint, $\rho_{+}(\infty)=\rho_{-}(\infty)+Z \rho_{\mathrm{p}}$, closes the problem [25]. The total concentration of microions inside the suspension is then related to their concentration inside the salt reservoir through

$$
(\kappa a)^{4}=\left(\kappa_{\mathrm{s}} a\right)^{4}+[12 \eta(1+\kappa a)]^{2} .
$$

The osmotic pressure, within the non-linear PB theory, is determined from the concentration of microions in the region where the electric field is zero. Since the electrostatic potential decays exponentially with $r$, the electric field vanishes when $r \rightarrow \infty$. Within the jellium approximation the osmotic pressure then takes a particularly simple form:

$$
\beta P=\rho_{+}(\infty)+\rho_{-}(\infty)=\frac{\kappa^{2}}{4 \pi \lambda_{\mathrm{B}}} .
$$

One can show that the solution of equation (28) obeys the inequality

$$
\left.\frac{\partial \kappa}{\partial \eta}\right|_{\kappa_{\mathrm{s}}}>0
$$


Table 1. The equation of state (25) with (third column) and without (fourth column) renormalization of volume terms, as a function of the electrostatic coupling $\lambda_{\mathrm{B}} / a$, for a packing fraction $\eta=0.00125$. The quantity $n$ denotes the mean total density of counterions $n=\rho_{\mathrm{p}} Z_{\text {bare }}$. The MC data (second column) are taken from [6]. Since the previous parameters do not correspond to the saturation regime of effective charges but only approach it, we have used the effective charge given by Alexander's prescription [18] to compute the pressure from equation (25) in the third column. The corresponding ratio $Z / Z_{\text {bare }}$ is indicated in the last column.

\begin{tabular}{lllll}
\hline$\lambda_{\mathrm{B}} / a$ & $\beta P / n, \mathrm{MC}$ & $\beta P / n, Z=Z_{\mathrm{eff}}$ & $\beta P / n, Z=Z_{\text {bare }}$ & $Z_{\text {eff }} / Z_{\text {bare }}$ \\
\hline 0.022 & 0.98 & 0.99 & 0.99 & 1.0 \\
0.044 & 0.95 & 0.96 & 0.97 & 0.99 \\
0.089 & 0.89 & 0.88 & 0.92 & 0.95 \\
0.178 & 0.71 & 0.68 & 0.78 & 0.82 \\
0.356 & 0.45 & 0.41 & 0.46 & 0.53 \\
0.712 & 0.26 & 0.21 & -0.32 & 0.29 \\
\hline
\end{tabular}

which ensures that the compressibility is always positive and that the suspension is stable against phase separation. In the absence of salt, we obtain a simple analytic expression:

$$
4 \pi \lambda_{\mathrm{B}} a^{2} \beta P=12 \eta[1+6 \eta+\sqrt{12 \eta(1+3 \eta)}],
$$

that will be tested against experimental data in section 4 .

\section{Discussion}

\subsection{Comparison with the MC simulations}

The above analysis shows that different routes to thermodynamic pressure lead to very different results. In order to decide which route is the most reliable, a comparison with 'exact results' is welcome. As a benchmark, we can use the MC pressure data of Linse [6] for salt free asymmetric electrolytes consisting of highly charged spherical macroions and point counterions. At high electrostatic couplings, this system exhibits an instability and separates into two coexisting phases of different electrolyte concentration. We shall argue that a minimum requirement for a reliable theory of phase behaviour is its ability to reproduce reasonably accurately the MC equation, at least up to the transition point. This appears to be a stringent test and a necessary condition for trusting any instability that a theory might predict.

We first test in table 1 the equation of state (25) for a charge asymmetry $Z_{\text {bare }}=40$ between colloids and counterions. It is evident that renormalization of colloidal charge significantly improves upon complete neglect of non-linearities. The latter approach consists in considering $Z=Z_{\text {bare }}$ and severely fails at high $\lambda_{\mathrm{B}} / a$, giving negative pressures. Our renormalized volume term captures the main effect of non-linearities, but the agreement with MC results, even if decent in view of the simplicity of the approach, is nevertheless only qualitative and does not reach the level of accuracy required for discussing phase stability.

The jellium equation of state derived in section 3 only holds for saturated effective charges, i.e. in a regime of coupling that the MC simulations, so far, have not reached (which corresponds to a very high bare charge with a large separation of scales between the Bjerrum length and colloid radius; see below). We therefore directly turn to the comparison of the relative performances of the PB cell model, symmetric PB theory [10] and bootstrap PB theory (see [11] for details), with respect to MC data (see figures 4 and 5).

To produce these figures (providing a similar comparison to that in table 1), we have chosen the lowest and the highest packing fractions investigated by Linse [6]. The striking 


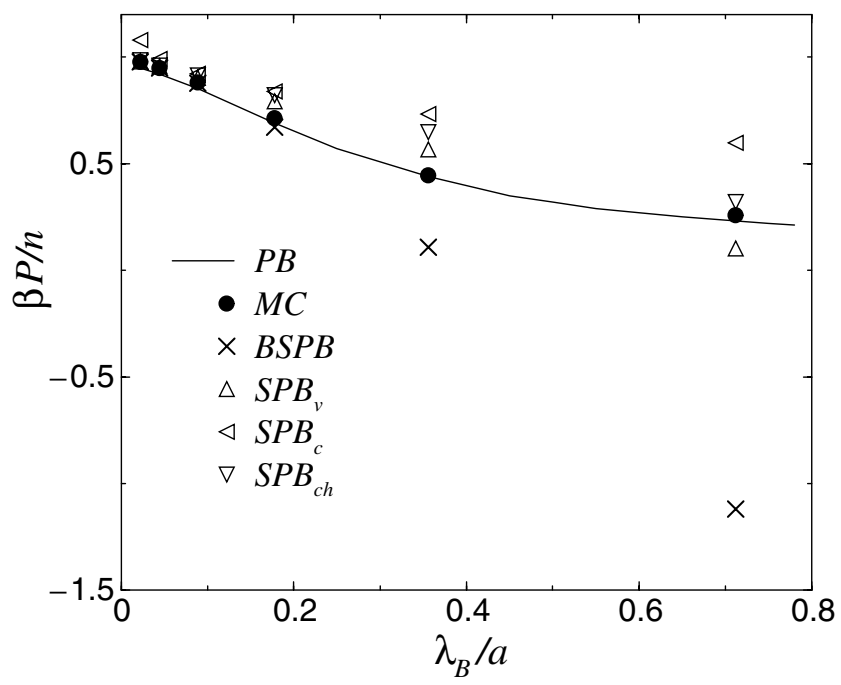

Figure 4. MC pressures (dots) compared to those obtained within the PB cell model (continuous curve), bootstrap PB theory [11] (crosses) and symmetric PB theory [10] (triangles, corresponding to virial, charging and compressibility routes). The packing fraction is $\eta=0.00125$ and $n=\rho_{\mathrm{p}} Z_{\text {bare }}$. As in table 1, the polyion/counterion charge asymmetry is $Z_{\text {bare }}=40$.

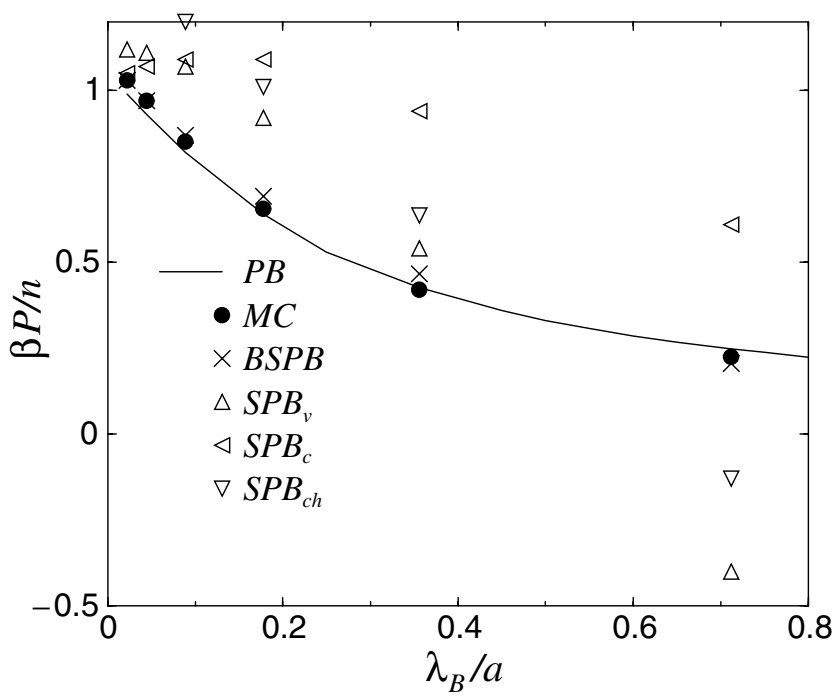

Figure 5. As figure 4, but for a packing fraction $\eta=0.08$.

feature revealed by figures 4 and 5 is the remarkably good agreement between the PB cell pressures [26, 27] and the MC simulations, even at $\eta=0.00125$ where the cell model could have been anticipated to fail (see also [28]). The only competitive approach at $\eta=0.08$ seems to be that of the bootstrap PB theory [11], but this theory severely fails for the low volume fractions - see figure 4-necessary for studying colloidal phase stability. At this volume fraction, the simple treatment of section 2 (equation (25)) provides a better equation of state than the bootstrap PB or symmetric PB theory (see table 1 and figure 4). Within the PB cell, 


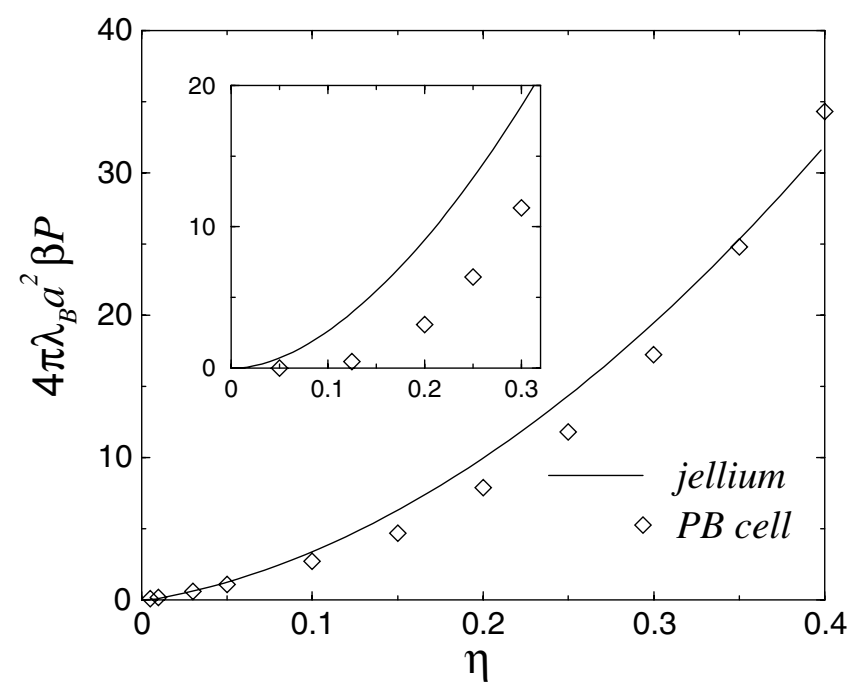

Figure 6. Comparison between PB cell pressures and those for the jellium model, without added salt (in the latter situation, the equation of state is given by expression (31)). Inset: the same when the suspension is dialysed against a salt reservoir such that $\kappa_{\mathrm{S}} a=2.6$ (the quantity $P$ considered is the osmotic pressure; i.e. the reservoir contribution has been subtracted).

polyion-polyion as well as counterion-counterion correlations are discarded; figures 4 and 5 show that as far as the pressure is concerned, these contributions are small or negligible, for the parameters investigated, even at the highest couplings. This justifies their neglect in our analytical treatment.

Given the accuracy of the PB cell model, we are now in a position to assess the quality of the jellium approximation of section 3. The corresponding pressures are compared in figure 6 with their PB cell counterparts for highly charged colloids (where the effective charge saturates to its upper threshold), both with and without added salt. The simple analytical expression (31) for salt free suspensions is found to be in good agreement with the PB data. Unfortunately the agreement deteriorates when $\kappa_{\mathrm{s}} a>1$ (see the inset).

\subsection{Relevance for colloidal suspensions}

At this point, we must conclude that the PB theory, even restricted to the cell, is superior to the competing approaches for aqueous suspensions with monovalent counterions. At high electrostatic couplings, corresponding to multivalent counterions in water, the MC simulations of Linse [6] find an instability. This transition, however, has nothing to do with the volume terms, but is the result of strong correlations between the double layers of colloidal particles [22] which produce attraction between like-charged colloids at sufficiently short separations [29, 30]. So far, this attraction has not been properly included in any of the thermodynamic theories of colloidal stability.

Validity of PB theory. To quantify the range of validity of the PB theory one may construct a dimensionless parameter $\Gamma_{\mathrm{cc}}$ characterizing the importance of microion correlations, discarded within the PB theory. For monovalent microions, $\Gamma_{\mathrm{cc}} \propto \beta q^{2} /(\ell \epsilon)$ where $\ell$ is the characteristic mean distance between the microions in the double layer. If the number of condensed counterions is such as to almost completely neutralize the colloidal charge, which is the case 
for strongly charged colloids, $\ell \simeq a / \sqrt{Z_{\text {bare }}}$ and $\Gamma_{\text {cc }}$ becomes $[22,29]$

$$
\Gamma_{\mathrm{cc}}=\frac{\lambda_{\mathrm{B}}}{a} \sqrt{\frac{Z_{\mathrm{bare}}}{4 \pi}} .
$$

When $\Gamma_{\mathrm{cc}}$ exceeds unity, PB theory is expected to break down. The value $\Gamma_{\mathrm{cc}} \simeq 2$ has been reported to correspond to the instability threshold [29, 31], which has been observed in the simulations of Linse [6]. The field theoretic treatment of Netz also corroborates this conclusion [32]. For particles with $Z_{\text {bare }}=40, \Gamma_{\mathrm{cc}} \simeq 2$ corresponds to $\lambda_{\mathrm{B}} / a \simeq 1.1$. Thus the PB theory can be expected to work quite well up to very high surface charge concentrations. Indeed, comparing the predictions of the PB cell model to those of the MC simulations, an excellent agreement is observed up to $\lambda_{\mathrm{B}} / a \simeq 0.7$ where the MC data stop, rather close to the expected point of instability $\lambda_{\mathrm{B}} / a \simeq 1.1$; see figures 4 and 5 .

Validity of the saturation picture within the PB theory. The constant potential approach used in sections 2 and 3 relies on the phenomenon of effective charge saturation exhibited by the PB theory when $Z_{\text {bare }}$ is large enough. The saturation occurs when the electrostatic energy of the condensed counterion is significantly larger than $k_{\mathrm{B}} T$. This can be characterized by a dimensionless parameter

$$
\Gamma_{\mathrm{sat}}=\frac{Z_{\mathrm{bare}}}{Z_{\mathrm{eff}}}=\frac{Z_{\mathrm{bare}} \lambda_{\mathrm{B}}}{4 a\left(1+\kappa_{\mathrm{s}} a\right)} .
$$

When $\Gamma_{\text {sat }}$ becomes larger than 1 [20,23], linearized theory fails and charge renormalization becomes important.

We must stress that large values of $\Gamma_{\text {sat }}$ are fully compatible with small values of $\Gamma_{\mathrm{cc}}$. Specifically, for any $a \gg \lambda_{\mathrm{B}}$, there exist a range of bare colloidal charges $Z_{\text {bare }}$ such that $\Gamma_{\mathrm{cc}}<2<\Gamma_{\text {sat }}$. For these values of $Z_{\text {bare }}$, the polyion-microion interaction is sufficiently strong that linearized theories, without charge renormalization, will certainly fail. On the other hand the counterion-counterion correlations are sufficiently weak that the PB theory is still applicable. To illustrate this point, we compare in figure 7 the pressure obtained at saturation within the PB cell model calculation (formally $Z_{\text {bare }} \rightarrow \infty$ ) to that measured experimentally by Reus et al, under conditions very close to complete deionization (no salt). The agreement with PB theory had already been mentioned in [20,33], but provides an illustration of the saturation phenomenon in real suspensions. It also shows that despite its simplicity, equation (31) is fairly accurate.

It is important to stress, however, that for any colloidal size $a$, there is a maximum value of $Z_{\text {bare }}$ above which the PB theory fails. In practice, however, this breakdown of the PB equation never occurs for aqueous suspensions containing only monovalent counterions. The reason for this is that the hydrated ionic size provides a lower cut-off for the length $\ell$ which appears in $\Gamma_{\mathrm{cc}}$, i.e. $\ell>d$, where $d \approx 4 \AA$ is the hydrated ionic diameter. For aqueous suspensions with monovalent counterions $\Gamma_{\mathrm{cc}}$ is, therefore, always less than $\lambda_{\mathrm{B}} / d$, so $\Gamma_{\mathrm{cc}}<2$. For monovalent counterions in water, the PB theory, therefore, should apply without any restriction. Of course, this pleasing situation changes as soon as multivalent salt is added to the suspension. In this case, hydrated ionic size is no longer sufficient to restrict the strength of microion-microion correlations and $\Gamma_{\mathrm{cc}}>2$. Under these conditions the PB theory will no longer apply and a more sophisticated approach must be used.

\section{Summary and conclusions}

We have proposed (section 2) a linear theory for investigating the phase behaviour of colloidal suspensions. The non-linear effects are partially taken into account through the postulate that 


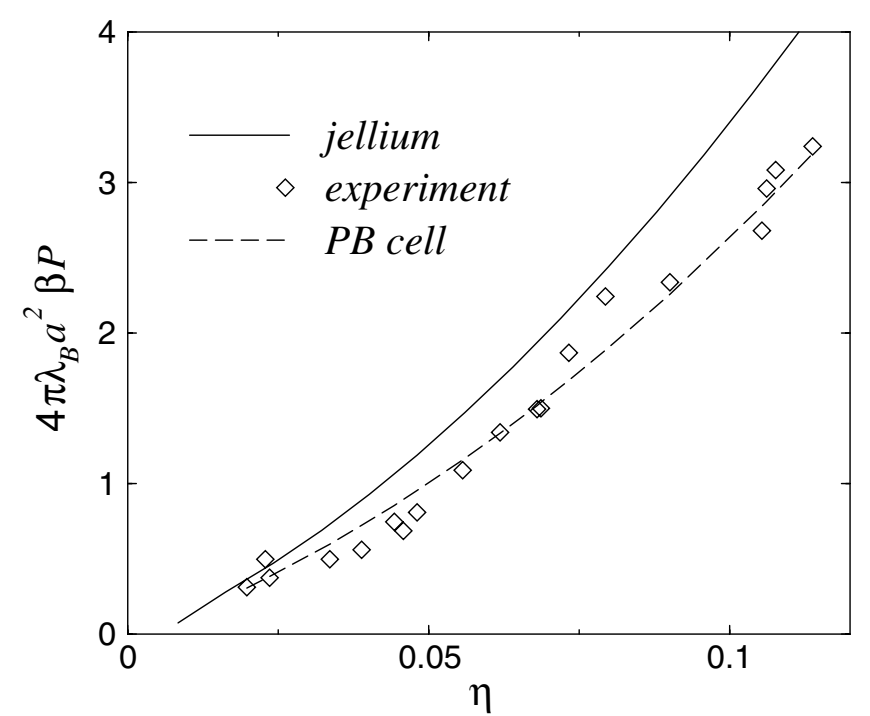

Figure 7. As figure 6, but including a comparison with osmotic pressures of deionized bromopolystyrene particles (shown by diamonds, from [33]). The curve labelled 'jellium' corresponds to the analytical prediction (31). No adjustable parameters have been used.

highly charged polyions behave as if they were constant potential objects. The effective charge of colloidal particles is, therefore, a state dependent function. Our first goal was to develop a consistent thermodynamic approach for such state dependent charges. The results found in section 2 rely on a simple form for the electrostatic free energy (volume term), resulting from the polyion-microion interactions calculated using the linearized PB equation, for spherical colloids. The approach could be easily generalized to the case of cylindrical macroions. The critical behaviour predicted is, however, spurious, which may be attributed to the simplicity of the volume term used and/or the difficulty of renormalizing such terms. The jellium-like model of section 3 provides a more reliable route and allows one to obtain analytically a simple equation of state for highly charged colloids in the salt free limit; see figure 7 . Unfortunately it is difficulty to see how this kind of approach can be extended to account for the polyion-polyion interactions.

From our analysis, we conclude that for $\Gamma_{\mathrm{cc}}=\left[Z_{\mathrm{bare}} /(4 \pi)\right]^{1 / 2} \lambda_{\mathrm{B}} / a<2$, the PB approach, even restricted to the cell model, leads to more accurate predictions for the thermodynamic functions than the competing theories. This is quite remarkable, since it is by far the simplest (see the appendix of [34] for a 'ready-to-use' implementation of the PB cell model). However, there is clearly a need to go beyond the PB theory when dealing with multivalent counterions, since it is the counterion-counterion correlations that drive a phase instability for $\Gamma_{\mathrm{cc}}>2$. Inclusion of these effects in a theoretical approach is a difficult task, since they have little signature in the pressure data up to the electrostatic coupling where suddenly they destabilize the system.

\section{Acknowledgments}

This work was supported in part by the Brazilian agencies CNPq and FAPERGS and by the French CNRS. YL acknowledges the hospitality of the Theoretical Physics Laboratory in Orsay where part of this work was performed. 


\section{References}

[1] van Roij R and Hansen J-P 1997 Phys. Rev. Lett. 793082

[2] Levin Y, Barbosa M C and Tamashiro M N 1998 Europhys. Lett. 41123

[3] Tamashiro M N, Levin Y and Barbosa M C 1998 Physica A 258341

[4] van Roij R, Dijkstra M and Hansen J-P 1999 Phys. Rev. E 592010

[5] Warren P 2000 J. Chem. Phys. 1124683

[6] Linse P 2000 J. Chem. Phys. 1134359

[7] Diehl A, Barbosa M C and Levin Y 2001 Europhys. Lett. 5386

[8] von Grünberg H H, van Roij R and Klein G 2001 Europhys. Lett. 55580

[9] Deserno M and von Grünberg H H 2002 Phys. Rev. E 66011401

[10] Bhuiyan L B and Outhwaite C W 2002 J. Chem. Phys. 1162650

[11] Petris S N, Chan D Y C and Linse P 2003 J. Chem. Phys. 1185248

[12] Téllez G and Trizac E 2003 J. Chem. Phys. 1183362

[13] Dufrêche J-F, White T O and Hansen J-P 2003 Mol. Phys. 101174

[14] Tamashiro M N and Schiessel H 2002 Preprint cond-mat/0210246

[15] Fisher M E and Fishman S 1981 Phys. Rev. Lett. 47421

[16] Belloni L 1993 J. Chem. Phys. 988080

[17] Belloni L 1998 Colloids Surf. A 140227

[18] Alexander S, Chaikin P M, Grant P, Morales G J, Pincus P and Hone D 1984 J. Chem. Phys. 805776

[19] Trizac E, Bocquet L and Aubouy M 2002 Phys. Rev. Lett. 89248301

[20] Bocquet L, Trizac E and Aubouy M 2002 J. Chem. Phys. 1178138

[21] Marcus R A 1955 J. Chem. Phys. 231057

[22] Levin Y 2002 Rep. Prog. Phys. 651577

[23] Aubouy M, Trizac E and Bocquet L 2003 J. Phys. A: Math. Gen. 365835

[24] Trizac E and Hansen J-P 1997 Phys. Rev. E 563137

[25] Trizac E, Aubouy M and Bocquet L 2003 J. Phys.: Condens. Matter 15 S291

[26] Wennerström H, Jönsson B and Linse P 1982 J. Chem. Phys. 764665

[27] A simple derivation may also be found in Deserno M and Holm C 2001 Electrostatic Effects in Soft Matter and Biophysics (NATO Science Series II: Mathematics, Physics and Chemistry vol 46) ed C Holm, P Kékicheff and R Podgornik (Dordrecht: Kluwer)

[28] Lobaskin V and Linse P 1999 J. Chem. Phys. 1114300

[29] Rouzina I and Bloomfield V A 1996 J. Phys. Chem. 1009977

[30] Levin Y 1999 Physica A 265432

[31] Linse P and Lobaskin V 2000 J. Chem. Phys. 1123917

[32] Netz R R 2001 Eur. Phys. J. E 5557

[33] Reus V, Belloni L, Zemb T, Lutterbach N and Versmold H 1997 J. Physique II 7603

[34] Trizac E, Bocquet L, Aubouy M and von Grünberg H H 2003 Langmuir 194027 